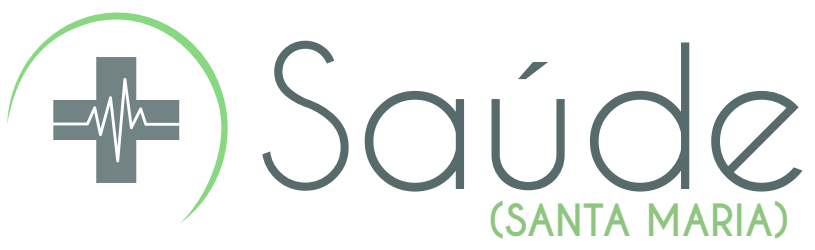

\title{
Cuidado farmacêutico aplicado a mulheres com câncer de mama na Atenção Primária à Saúde
}

\section{Pharmaceutical care applied to women with breast cancer in Primary Health Care}

\author{
Fernanda Fávero Alberti, Manuela Borges Sangoi Cardoso, Liana Pedrolo \\ Canterle, Eduardo Kraetzig Donini
}

\section{RESUMO}

O cuidado farmacêutico é uma ferramenta de acompanhamento farmacoterapêutico amplamente utilizada em diversos cenários da saúde. $\mathrm{O}$ objetivo desta pesquisa foi realizar o acompanhamento farmacoterapêutico de pacientes mulheres pertencentes a um grupo de apoio ao câncer de mama - Grupo Ellas -, vinculado à Atenção Primária à Saúde de um município da região centro-oeste do estado do Rio Grande do Sul. A metodologia de acompanhamento foi realizada conforme o Método Dáder. Foram realizadas 18 intervenções não-farmacológicas para as pacientes do estudo, após serem submetidas há uma média de três consultas farmacêuticas com cada paciente. A média de idade das participantes da pesquisa foi de 54,1 ( $\pm 8,2)$ anos. A média de menopausa das pacientes era de 44,4 ( $\pm 8,2)$ anos e a idade de menarca de 13,2 ( $\pm 2,0)$ anos. Foram identificados três problemas relacionados à medicamentos e nove resultados negativos à medicamentos. Em concordância ao objetivo proposto, verificou-se o quão importante é instituir na prática farmacêutica as ferramentas de cuidado e acompanhamento farmacoterapêutico em diferentes cenários clínicos.

Descritores: Acompanhamento farmacoterapêutico; Farmácia Clínica; Pacientes oncológicos.

Como citar este artigo: Alberti FF, Cardoso MBS, Canterle LP, Donini EK. Cuidado farmacêutico aplicado à mulheres com câncer de mama na atenção primária à saúde. Revista Saúde (Sta. Maria). $2018 ; 44(1): 1-8$

Autor correspondente: endereço não informado pelos autores

Data de Submissão: 11/09/2017

Data de aceite: $26 / 03 / 2018$

Conflito de Interesse: Não há conflito de interesse

\section{(c) $)$ BY-NC-ND}

\begin{abstract}
Pharmaceutical care is a pharmacotherapeutic monitoring tool widely used in various health settings. The objective of this research was to perform the pharmacotherapeutic follow-up of female patients belonging to a breast cancer support group - Ellas Group -, linked to the Primary Health Care of a city in the central-western region of the state of Rio Grande do Sul. followup methodology was performed according to the Dáder Method. Eighteen non-pharmacological interventions were performed for the study patients, after being submitted there are an average of three pharmaceutical consultations with each patient. The mean age of the study participants was 54.1 years $(S D=8.2)$. The mean age of menopause was 44.4 years $(S D=8.2)$ and the age of menarche was 13.2 years $(S D=2.0)$. Three drug-related problems and nine negative drug outcomes were identified. In agreement with the proposed objective, it was verified how important it is to institute in the pharmaceutical practice the tools of care and pharmacotherapeutic monitoring in different clinical scenarios.
\end{abstract}

Keywords: Pharmacotherapeutic follow-up; Clinical Pharmacy; Cancer patients. 


\section{Introdução}

O cenário oncológico representa, nos tempos atuais, um grande problema de saúde pública e impõe aos gestores, profissionais e trabalhadores do Sistema Único de Saúde (SUS) o desafio da integralidade do cuidado, isto é, inserir de forma abrangente propostas de intervenção de prevenção, proteção e recuperação da saúde das pessoas. Por ser considerada uma condição clínica crônica, o câncer é uma das doenças mais temidas do século XXI, caracterizando a necessidade de estratégias eficientes, que ultrapassem o viés interdisciplinar e consigam dialogar, de uma forma geral, com todos os profissionais de saúde ${ }^{1}$.

No que se refere ao câncer de mama, é o tipo de câncer com maior assiduidade e constância entre a população feminina e, por isso, o mais temido. Caracteriza-se, segundo autores ${ }^{2}$ como um tumor maligno que se desenvolve através de alterações gênicas das células do ducto e do glóbulo mamário e necessita de abordagens terapêuticas mais específicas e agressivas para combatê-lo.

Nesse sentido, estudos evidenciam que a utilização da Atenção Farmacêutica como ferramenta para a prática do cuidado apresenta, dentre os principais benefícios, a diminuição de reações adversas e a melhoria da adesão ao tratamento farmacológico ${ }^{3-5}$. Neste contexto, o cuidado farmacêutico atuante dentro das equipes de saúde representa, ao paciente oncológico, uma mudança no cenário da integralidade do cuidado, essencialmente nas condições crônicas, caminhando para a melhoria dos resultados terapêuticos, particularmente no nível dos cuidados que compete a Atenção Primária à Saúde6.

Dessa forma, é evidente a necessidade de instituir a prática do cuidado farmacêutico, em especial ao paciente oncológico, de modo que sirva como uma ferramenta que possibilite a melhoria da condição clínica desses pacientes. Portanto, objetivou-se realizar o acompanhamento farmacoterapêutico de pacientes mulheres pertencentes a um grupo de apoio ao câncer de mama - Grupo Ellas -, vinculado à Atenção Primária à Saúde de um município da região centrooeste do estado do Rio Grande do Sul. Como objetivos específicos, têm-se: identificar Problemas Relacionados à Medicamentos (PRM) e Resultados Negativos a Medicamentos (RNM).

\section{Metodologia}

Trata-se de um estudo longitudinal prospectivo, realizado no Centro Materno Infantil (CMI) e no Centro de Práticas Profissionais (CEPP) da Universidade Regional Integrada do Alto Uruguai e das Missões, ambos localizados em um município da região centro-oeste do estado do Rio Grande do Sul, no período de março a julho de 2017. A população de estudo foi constituída por 16 mulheres que estivessem em tratamento para o câncer de mama e/ou que estivessem sob acompanhamento médico após tratamento curativo da doença.

A coleta de dados foi realizada de acordo com o Manual de Acompanhamento Farmacoterapêutico "Método Dáder ${ }^{77}$ desenvolvido pela Universidade de Granada/Espanha, que consiste em sete etapas: oferta do serviço, entrevista farmacêutica, primeiro estado de situação, fase de estudo, fase de avaliação, fase de intervenção e entrevistas sucessivas.

Esse trabalho está inserido dentro do projeto "Aplicação do processo de Atenção Farmacêutica em diferentes grupos populacionais", e, portanto, foi submetido anteriormente ao Comitê de Ética em Pesquisa com Humanos desta universidade, aprovado sob o Certificado de Apresentação para Apreciação Ética (CAAE) número 52744416.3.0000.5353, de acordo com a Resolução 466/20128. As entrevistas foram realizadas individualmente após assinatura do Termo de Consentimento Livre e Esclarecido (TCLE), e as informações transcritas para o formulário de seguimento farmacoterapêutico.

A análise dos dados foi realizada através do teste de Kolmogorov-Smirnov ${ }^{9}$ para verificar a distribuição Gaussiana. Variáveis contínuas com distribuição normal foram apresentadas em média \pm desvio padrão. Variáveis contínuas que não apresentaram distribuição Gaussiana foram expressas em mediana e intervalo interquartil. As variáveis categóricas foram descritas em frequências e porcentagens. As análises foram realizadas no software GraphPad Prism® versão 4.00 para Windows 29 (GraphPad Software, San Diego, CA). Alguns resultados também foram apresentados de maneira descritiva. 


\section{RESULTADOS:}

A população do estudo foi composta por 16 mulheres participantes do Grupo Ellas, atendidas pelo Centro Materno Infantil do município. Destas, 93,8\% ( $n=15$ ) eram portadoras de carcinoma ductal invasivo (CDI) e apenas uma participante possuía câncer abdominal com metástase na mama. Estipulou-se o total de 16 participantes na amostra, sendo 15 pacientes com câncer de mama: destas, 66,7\% ( $n=10)$ tiverem CDI na mama esquerda (ME) e 26,7\% ( $n=4)$ na mama direita (MD). E uma paciente possuía a particularidade de ter sofrido CDI nas duas mamas, em momentos distintos. Foi realizada uma média de três consultas farmacêuticas com cada paciente.

Conforme a Tabela 1, é possível observar as características gerais das pacientes:

Tabela 1. Características gerais das pacientes do estudo $(n=16)$

\begin{tabular}{ll}
\hline Idade (anos) & $54,1 \pm 8,2$ \\
Hipertensão (\%) & 43,7 \\
Pressão arterial sistólica $(\mathrm{mmHg})$ & $121,9 \pm 17,2$ \\
Pressão arterial diastólica $(\mathrm{mmHg})$ & $69,4 \pm 18,0$ \\
Tabagismo (\%) & 12,5 \\
IMC (kg/m²) & $24,0 \pm 3,4$ \\
Prática de atividades físicas (\%) & 68,8 \\
\hline
\end{tabular}

Fonte: Elaborado pela autora com base nos dados obtidos.

Na Tabela 2 é possível verificar as características clínicas das pacientes do estudo, com relação ao tratamento e fatores de risco. Os dados expressam as características clínicas de todas as pacientes, não diferenciando quem realiza ou já realizou tratamentos específicos.

Tabela 2. Variáveis associadas à patologia: tratamento e fatores de risco

$\begin{array}{ll}\text { Quimioterapia (\%) } & 87,5 \\ \text { Terapia Hormonal (\%) } & 75,0 \\ \text { Radioterapia (\%) } & 56,2 \\ \text { Histórico de CA na família (\%) } & 68,7 \\ \text { Metástase (\%) } & 25,0 \\ \text { Sessões de radioterapia (n) } & 31,9 \pm 14,8 \\ \text { Idade de menopausa (anos) } & 44,4 \pm 8,2 \\ \text { Idade da menarca (anos) } & 13,2 \pm 2,0 \\ \text { Tempo de tratamento hormonal (anos) } & 7,6 \pm 5,1\end{array}$

Fonte: Elaborado pela autora com base nos dados obtidos.

Verificou-se que existe uma tendência de menarca mais tardia com a descoberta do câncer de mama também mais tardia, embora não haja correlação significativa.

O tipo de tratamento mais utilizado, pela maioria das pacientes, foi a quimioterapia, frequente em $87,5 \%$ dos casos. Infelizmente não foi possível ter acesso aos prontuários e prescrições, por motivos de a maioria das pacientes já ter realizado as suas quimioterapias há alguns anos. Assim, o número de sessões de quimioterapia variava de oito a 24 sessões, divididas entre "quimioterapia branca" e "quimioterapia vermelha", conforme as próprias pacientes classificavam.

Logo após a quimioterapia, em segundo lugar, a terapia hormonal correspondeu a $75,0 \%$ do protocolo terapêutico das pacientes, sendo a mais prescrita, geralmente, antes ou após todas as sessões de quimioterapia. 0 principal medicamento utilizado foi o Tamoxifeno, com a dosagem de $20 \mathrm{mg}$.

Aradioterapia foi prescrita em $56,2 \%$ dos casos, caracterizada como terapia adjuvante ao tratamento quimioterápico. 
Por se tratar de uma abordagem local em um período de tempo mais reduzido, necessita de um maior número de sessões. Em média, foram realizadas 31,9 sessões. Também, não foi possível obter acesso aos protocolos radioterápicos realizados pelas pacientes em detrimento do tempo de tratamento já ter sido ultrapassado.

Das pacientes participantes do estudo, $60 \%$ relataram ter feito mastectomia para retirada completa da mama afetada e 33,3\% realizaram cirurgia do quadrante e/ou nas regiões axilares. Todas as mulheres que realizaram a cirurgia, seja por mastectomia ou do quadrante, apresentavam dificuldades funcionais e físicas no braço correspondente a mama afetada.

Na tabela 3, pode-se observar a prevalência do estadiamento I em mulheres com idade superior a 43 anos:

Tabela 3. Estágio do tumor conforme o sistema TNM - Classificação Internacional de Tumores da União Internacional Contra o Câncer - UICC

\begin{tabular}{ll}
\hline Estadiamento & $(\%)$ \\
\hline Estágio 0 & 0 \\
Estágio I & 6,2 \\
Estágio II & 12,5 \\
Estágio III & 18,7 \\
Estágio IV & 25 \\
Desconhecem & 37,5 \\
\hline
\end{tabular}

Fonte: Elaborado pela autora com base nos dados obtidos.

Na figura abaixo é possível visualizar uma correlação positiva entre o estadiamento do câncer e a metástase, representada em $25,0 \%$ dos casos.

Figura 1. Correlação entre metástase e estadiamento.

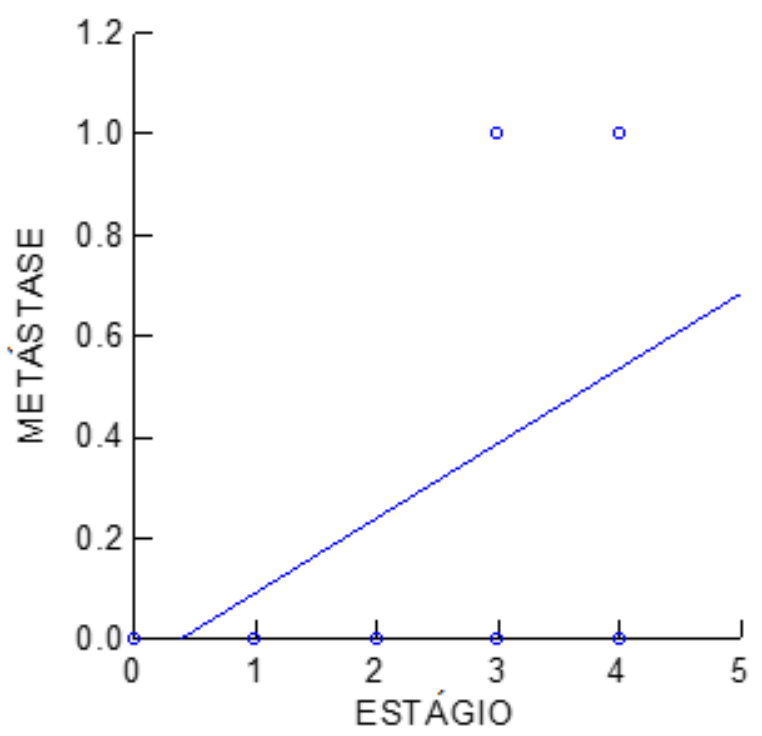

Fonte: Elaborado pela autora com base nos dados obtidos.

De uma forma geral, em 100,0\% dos casos de metástase, 36,0\% são explicados pelo estágio do câncer de mama.

Foram identificados três Problemas Relacionados à Medicamentos (PRM), sendo dois no eixo da necessidade (PRM 1) e um no eixo da efetividade (PRM 4). Falhas na farmacoterapia, categorizadas dentro da Farmácia Clínica como PRM e Resultados Negativos a Medicamentos (RNM), sujeitam o paciente a uma redução drástica da sua qualidade de vida, tendo como a principal consequência o agravo decorrente do uso de medicamentos, podendo chegar ao óbito ${ }^{10}$. 
Observou-se a prevalência dos seguintes fármacos: Hidroclorotiazida (25\%), Ácido ascórbico (vitamina C) (12,5\%), Levotiroxina sódica (12,5\%), Sulfato de glucosamina (12,5\%) e Captopril (12,5\%). Essas medicações foram utilizadas para o tratamento decorrente de outras doenças, como Hipertensão Arterial Sistêmica (HAS) e, também, para o tratamento de complicações adversas da terapêutica empregada para o tratamento do câncer, como é o caso da vitamina C e do carbonato de cálcio.

Não foram detectadas interações medicamentosas significativas, visto que a maioria das pacientes utilizava até dois medicamentos concomitantemente ou, ainda, havia aquelas que não faziam uso de nenhuma medicação.

Foram identificados oito RNMs, condizentes com a situação clínica de cada paciente, expressos abaixo:

Tabela 4. Resultados negativos a medicamentos verificados

\begin{tabular}{ccl}
\hline RNM & EIXO & DEFINIÇÃO DO PROBLEMA \\
\hline$n=6$ & Segurança & Insegurança não quantitativa \\
$n=1$ & Efetividade & Inefetividade quantitativa \\
$n=1$ & Necessidade & Problema de saúde não tratado \\
\hline
\end{tabular}

Fonte: Elaborado pela autora com base nos dados obtidos.

Observou-se que houve seis pacientes em que a predominância de RNM foi relacionada à segurança do medicamento prescrito que independe da dosagem, sendo o caso de efeito relatado pelo uso do Tamoxifeno $(n=5)$ e do Captopril $(n=1)$.

\section{Discussão}

Observou-se que o desenvolvimento do câncer de mama possui relação com a idade e o histórico familiar da mulher, havendo estudos nos quais se pode observar que a sua prevalência aumenta rapidamente até os 50 anos de idade $^{12}$. O rastreamento mamográfico, neste contexto, é necessário para reduzir a mortalidade de mulheres suscetíveis ao câncer de mama, realizando um diagnóstico precoce em caso de incidência de sintomas. Estudo afirma que esse rastreamento pode reduzir em até $18 \%$ o número de mortes por câncer de mama nas mulheres com idade entre 40 e 50 anos. Adicionalmente, após os 50 anos idade, a redução supera $30 \%{ }^{13}$.

A idade é fator primordial para definição do prognóstico da doença. Alguns estudos delinearam que mulheres com idade entre 40 e 50 anos tem um melhor prognóstico de vida, enquanto que mulheres com idade inferior aos $30 \mathrm{e}$ acima dos 75 anos possuem uma taxa de sobrevida menor. $O$ estadiamento clínico é considerado um fator importante se levarmos em conta que essa faixa etária com prognóstico satisfatório geralmente tem o seu diagnóstico confirmado de forma precoce do que as demais ${ }^{14-15 .}$

Não foi observada uma relação direta do câncer de mama com doenças como DM e/ou HAS neste estudo. No entanto, alguns autores presumem que em razão de o câncer de mama ser desencadeado por um longo processo fisiológico, com agravantes de inúmeros fatores de risco, é possível que haja sim uma tendência. De acordo com autores16, as pessoas possuem o conhecimento das associações entre tabagismo e câncer, sedentarismo e infarto agudo do miocárdio, alimentação inadequada e diabetes. Entretanto, não conseguem assimilar a existência de uma correlação entre sedentarismo, tabagismo, alimentação inadequada e o câncer, o que demonstra o desconhecimento da cadeia dos fatores de risco que podem desencadear a doença.

Quanto à menarca, certo autor ${ }^{17}$ descreve que, em comparação a meninas que tiveram a primeira menstruação aos 16 anos, meninas que iniciaram a menarca dois ou três anos antes apresentaram um risco de $10 \%$ a $30 \%$ maior de desenvolver a doença ao longo dos anos. Com isso, pode-se inferir que a idade média de menarca das pacientes 
representa um agravante em sua condição clínica, possivelmente oriundo de características fisiológicas, hormonais e/ ou genéticas de seus antecedentes.

Com relação à menopausa, o normal do processo fisiológico é o cessamento da menstruação entre os $45 \mathrm{e}$ 55 anos de idade. No entanto, mulheres que iniciam a menopausa após os 55 anos possuem $50 \%$ mais chances de desenvolvimento do câncer de mama. Nesse sentido, a menopausa tardia gera a exposição prolongada ao estrogênio e, após os 53 anos de idade, caracteriza sério risco para o desenvolvimento do câncer de mama ${ }^{18}$. Considerando isto, salvo algumas exceções, todas as pacientes estavam dentro dos padrões considerados fisiologicamente normais.

Todas as pacientes que estavam realizando tratamento hormonal utilizavam do fármaco Tamoxifeno. Vários estudos demonstram e elucidam o seu mecanismo de ação como forma de redução do câncer mamário e aumento da sobrevida das pacientes19,20. 0 tempo de tratamento hormonal variou de acordo com o estadiamento dos tumores das pacientes. Em resumo, a variação encontrada foi de 05 a 07 anos de administração do medicamento por via oral. Evidentemente, foram relatadas muitas situações de potencial não adesão ao tratamento farmacológico com o uso de Tamoxifeno, devido ao longo período de exposição e aos seus efeitos adversos.

$\mathrm{Na}$ visão das pacientes, a radioterapia representa um grande desafio a ser superado, pois se trata de um protocolo dificultoso em termos de postura e nenhum pouco agradável quando a sua realização. Conforme autor, o tratamento primário é a intervenção cirúrgica com o principal objetivo de retirar o câncer. Além disso, esse procedimento possibilita a retirada também de outras áreas afetadas ${ }^{21}$.

Foi observado que a maioria das pacientes que realizaram algum procedimento cirúrgico na mama sofriam de dores fortes nos braços. Segundo autores é recomendado que após a cirurgia não seja realizado nenhum tipo de movimento que possa causar infecções no membro pois essa dificuldade funcional é característica de linfedema ${ }^{22}$.

Foi elucidada uma correlação entre metástase e estadiamento. Essa associação ainda não é bem descrita na literatura, mas se reconhece, por diversos autores, a tendência maléfica do prognóstico de pacientes com câncer de mama localmente avançado (estadiamento III e IV) 17,23.

As intervenções farmacêuticas realizadas proporcionaram às pacientes uma forma diferenciada e uma alternativa eficaz no processo de autocuidado à saúde. Em estudo realizado por autores, foi demonstrado que as intervenções farmacêuticas voltadas à reeducação dos pacientes são importantes para que estes se sintam mais preparados para lidar com os efeitos adversos que os tratamentos proporcionam ${ }^{24}$.

\section{Conclusão}

O presente estudo contribuiu de forma significativa na oferta de cuidado que implicasse diretamente na melhora da condição clínica de mulheres com câncer de mama nos diversos aspectos da integralidade do cuidado. A implementação de um serviço de Clínica Farmacêutica, voltado para pacientes com essas condições crônicas, representa um grande avanço para a profissão farmacêutica, impactando diversos cenários, entre eles a atenção primária à saúde.

Os serviços de atenção primária demandam de profissionais qualificados principalmente no atendimento de pacientes oncológicos. Como a maioria dos tratamentos são realizados na alta complexidade, sem que haja o compartilhamento do cuidado com serviços da atenção primária à saúde, fato que implica na descontinuidade do cuidado quando os pacientes retornam a seus domicílios. Nesse sentido, ficou evidente a caracterização dessa necessidade na ampla receptividade que este estudo obteve por parte das participantes ao relatarem suas angústias, medos e sofrimentos. 0 profissional farmacêutico deve se qualificar para atuar neste cenário, corroborando com a implementação da Farmácia Clínica e com estudos exitosos visando o bem-estar do paciente.

Aidentificação de PRMs e RNMs determinou a abordagem a ser realizada às pacientes, sendo de suma importância para a sua conscientização quanto ao uso racional e hábitos apropriados de administração de medicamentos, além de fornecer informações importantes para a realização dos seus respectivos tratamentos. As intervenções foram o subsídio que permitiu indicar que a prática clínica é fundamental na determinação da melhor abordagem possível para a melhora da condição clínica das pacientes.

Entretanto, este estudo teve limitações no decorrer da aplicação do método Dáder, em virtude da falta de acesso às outras informações pertinentes à caracterização do processo saúde-doença das participantes, como, por exemplo, o os prontuários médicos e prescrições dos serviços especializados no tratamento quimioterápico e radioterápico. Isso, 
na prática, impossibilitou uma avaliação terapêutica mais aprofundada, voltada realmente à determinação de uma farmacoterapia efetiva, segura e necessária.

Em concomitância aos objetivos propostos pelo estudo, considera-se essencial a atuação clínica do profissional farmacêutico e a implementação deste serviço, prioritariamente, na atenção primária à saúde dos Municípios, considerando os aspectos que regem a integralidade do cuidado e o aporte teórico-prático fornecido por este profissional.

\section{Referências}

1. Instituto Nacional do Câncer José Alencar Gomes da Silva (INCA). ABC do câncer: abordagens básicas para 0 controle do câncer. 2a ed. Rio de Janeiro/RJ: INCA; 2011.

2. Smeltzer SC, Bare GB, Brunner \& Suddarth. Tratado de Enfermagem Médico- Cirúrgica. 10a ed. Rio de Janeiro: Guanabara Koogan; 2005.

3. Weidle P, Bradley L, Gallina J, Mullins CD, Thorn D, Siegel LP. Pharmaceutical care intervention documentation program and related cost savings at a university hospital. Lippincott's Hospital Pharmacy. 1999; 34(1): 43-52.

4. Narhi U, Airaksinen M, Tansknanen P, Erlund $H$. Terapeutic outcomes monitoring by community pharmacists for improving clinical outcomes in asthma. J. Clin. Pharm. Ther. 2000; 25(3):177-83.

5. Bernsten C, Bjorkman I, Caramona M, Crealey G, Frokjaer B, Rundberger E. Improving the well-being of elderly patients via community pharmacy-based provision of pharmaceutical care: a multicentre study in seven Europcan countries. Drug Aging. 2001; 18(1):63-77.

6. Ministério da Saúde (BR). Secretaria de Ciência, Tecnologia e Insumos Estratégicos. Departamento de Assistência Farmacêutica Insumos Estratégicos. Serviços farmacêuticos na atenção básica à saúde. Brasília: Ministério da Saúde, 2014.

7. Hernández DS, Castro MMS, Dáder MJF. Método Dáder: manual de seguimento farmacoterapêutico. 3a ed. Grupo de Investigación en Atención Farmacéutica (CTS-131). Granada (Espanha): Universidad de Granada; 2014.

8. Conselho Nacional de Saúde (Brasil). Resolução nº. 466, de 12 de dezembro de 2012. Brasília; 2012.

9. Fernandes FS. Testes de Ajuste a Distribuições Estatísticas e Métodos para Estimação dos Parâmetros em Análises de Fiabilidade [dissertação]. Lisboa (Portugal): Instituto Superior de Engenharia de Lisboa; 2013.

10. Mota DM, Silva MGC, Sudo EC, Ortún V. Uso racional de medicamentos: uma abordagem econômica para tomada de decisões. Ciênc. saúde coletiva [Internet]. 2008 Apr [cited 2018 Mar 22]; 13(Suppl): 589-601.

11. Santos HJ, Iglésias-Ferreira P, Ribeiro PL, Nunes-da-Cunha I. Introdução ao seguimento farmacoterapêutico. Lisboa (Portugal): Universidade Lusófona; 2007.

12. Smith RA, D'Orsi CJ. Screening for breast cancer. In: Harris Jr., Lipmann ME, Morrow M, Osborne CK, editors. Diseases of the breast. Philadelphia: Lippincott; 2005. p.103-30.

13. Host H, Lund E. Age as a prognostic factor in breast cancer. Cancer. 1986; 57: 2217-21. Errata em: Cancer, 1986; 58(4):996.

14. Santi M, Capocaccia R, Verdecchia A, Esteve J, Gatta G, Micheli A. Survival of women with breast cancer in Europe: variation with age, year of diagnosis and country. Int J Cancer. 1998; 77:679-83. 
15. Abreu E, Koifman J. Fatores prognósticos no câncer de mama feminino. Rev. Brasileira de Cancerologia. 2002; 1(48):113-31.

16. Borges TT, Rombaldi AJ, Knuth AG, Hallal PC. Conhecimento sobre fatores de risco para doenças crônicas: estudo de base populacional. Cad. Saúde Púb. 2009; 25(7):1511-1520.

17. Vogel VG. Breast cancer prevention: a review of current evidence. J. Clinic. Oncol. 2000; 50(3):156-70.

18. Toniolo PG, Levitz M, Zeleniuch-Jacquotte A, Banerjee S, Koenig KL, Shore RE. A prospective study of endogenous estrogens and breast cancer in post menopausal women. J Natl Cancer Inst. 1995; 87(3):190.

19. Moerkens M, Zhang Y, Wester L, Van de Water B, Meerman JH. Epidermal growth factor receptor signalling in human breast cancer cells operates parallel to estrogen receptor a signalling and results in tamoxifen insensitive proliferation. BMC Cancer. 2014; 1(14):283.

20. Zapaterini JR. Avaliação histopatológica e molecular da imunoterapia com p-mapa associada ao tamoxifeno na progressão do câncer de mama induzido quimicamente em ratas Sprague-Dawley [dissertação]. São Paulo: Universidade Estadual Paulista "Júlio de Mesquita Filho" Câmpus de Botucatu; 2017.

21. Silva LC. Câncer de mama e sofrimento psicológico: aspectos relacionados ao feminino. Psico. Est. 2008; 13(2): $231-237$

22. Cantinelli FS et al. A oncopsiquiatria no câncer de mama - considerações a respeito de questões do feminino. Rev. de Psiquiatria Clínica. 2006; 3(33):124-133.

23. Henson DE, Ries L, Freedman MA, Carriaga M. Relationship among outcome, stage of disease, and histologic grade for 22.616 cases of breast cancer. Cancer; 1991. 68:2142-2149.

24. Simons S, Ringsdorf S, Braun M. Enhancing adherence to capecitabine chemotherapy by means of multidisciplinary pharmaceutical care. Supportive Care in Cancer; 2011. 7(20):1009-1018. 